\title{
Coronary Artery Dissection in the Left Main Treated with Stenting in a Patient with Multiple Re-infarction - Case Report
}

\author{
Nyulas Tiberiu $^{1 \star}$, Blendea Ciprian ${ }^{1,2}$, Barcan Andreea ${ }^{1}$, Bajka Balazs ${ }^{1,2}$, Korodi Szilamer $^{1}$, Rat Nora ${ }^{1}$ \\ 1 University of Medicine and Pharmacy of Tîrgu Mureș, Clinic of Cardiology, Tîrgu Mureș, Romania \\ ${ }^{2}$ Cardio Med Medical Center, Department of Advanced Multimodal Imaging, Tîrgu Mureș, Romania
}

Introduction: In complex cases of multiple coronary artery stenosis, revascularization strategy could be essential for improving the life expectancy andqualityof life. However, major complications are sometimes encountered during interventions, such as rupture of the atheromatous plaque with consequent dissection of the coronary artery, causing an acute coronary syndrome which requires immediate intervention from the operator. In the absence of an experienced interventional cardiologist a complication like this can be fatal. Case presentation: We present the case of a 67-years old patient, male, with a known history of cardiovascular disease, who presented in our service complaining of chest pain with tightening character, irradiation in the shoulder and left arm, respectively shortness of breath and fatigue. The patient presented a history of multiple infarctions, intervention and stenting on RCA and circumflex artery. Computed Tomographic Coronary Angiography provided detailed information on the location of the target lesions and was followed by a revascularization procedure. However, despite the complex pre-interventional assessment, while trying to engage the guide in the emergence ofthe circumflex artery, atherosclerotic plaque rupture occurred, causing a dissection of the coronary wall which extended retrogradely into the left main, requiring a rapid response from the operator. A coronary stent was implanted into the left coronary artery trunk, treating the dissection. Conclusions: Coronary artery dissection is a very serious complication that can occur during a complex revascularization procedure, requiring immediate intervention in order to save the patient's life.

Keywords: atheroma, revascularization, dissection

Received: 25 September 2015 / Accepted: 06 November 2015

\section{Introduction}

Spontaneous coronary artery dissection is a very rare cause of acute coronary syndromes in young otherwise healthy patients with a striking predilection for the female gender. It was reported for the first time in 1931 at the autopsy of a 42-year-old woman [1]. Pretty published the first case of coronary artery dissection [1], while Forker reported the first angiographic diagnosis of such a case [2]. However, coronary artery dissection is encountered more frequent during a revascularization procedure and can put the life of the patient at a significant risk. We present the case of a patient with multiple revascularization procedures in the history, who presented a dissection of the left coronary ostium during a percutaneous coronary intervention.

\section{Case report}

Patient aged 67, male, with a known history of cardiovascular disease, presented in our service complaining of chest pain with tightening character, irradiating in the shoulder and the left arm, accompanied by shortness of breath and progressive fatigue. The patient was diagnosed in 1999 with inferior myocardial infarction, which was treated with implantation of two stents, one in the right coronary artery and one in the circumflex artery. In 2013, the patient suffered another inferior myocardial infarction,

* Correspondence to: Tiberiu Nyulas

E-mail: tiberiu.nyulas@gmail.com which was thrombolysed but without signs of reperfusion, therefore the patient underwent a coronary angiography which showed occlusion of the right coronary artery, therefore another stent was implanted in the right coronary artery. In 2015, the patient presented again to the hospital complaining of chest pain with a burning character. ECG at admission indicated pathological $Q$ waves in the inferior leads, flat T waves in DI, DII, DIII and V6 (figure 1). Echocardiography at admission revealed an atheromatous aortic wall, first degree mitral regurgitation, first degree tricuspid regurgitation, ejection fraction of $48 \%$ and a septal and side wall hypokinesia. Blood counts and renal function were normal. AngioCT examination was performed for a better evaluation of the coronary bed and indicated the right coronary artery without significant stenosis and a permeable stent, without any sign of in-stent restenosis (figure 2). In figure 3 we can see the circumflex artery stent restenosis and the occlusion located proximal to the stent, revealed by Angio CT. Also, we can see calcified plaques in the anterior descending artery. The occlusion proximal to the stent and the stent restenosis can be viewed in detail in figure 4 . The examination of the coronary arteries with coronarography confirmed the occlusion of the circumflex artery at its origin from the left main, proximal to the first stent, therefore a revascularization procedure was decided. At the first attempt to cross the occlusion with a BMW guide, a coronary artery dissection was produced caused by the rupture of the atherosclerotic plaque, which progressed 


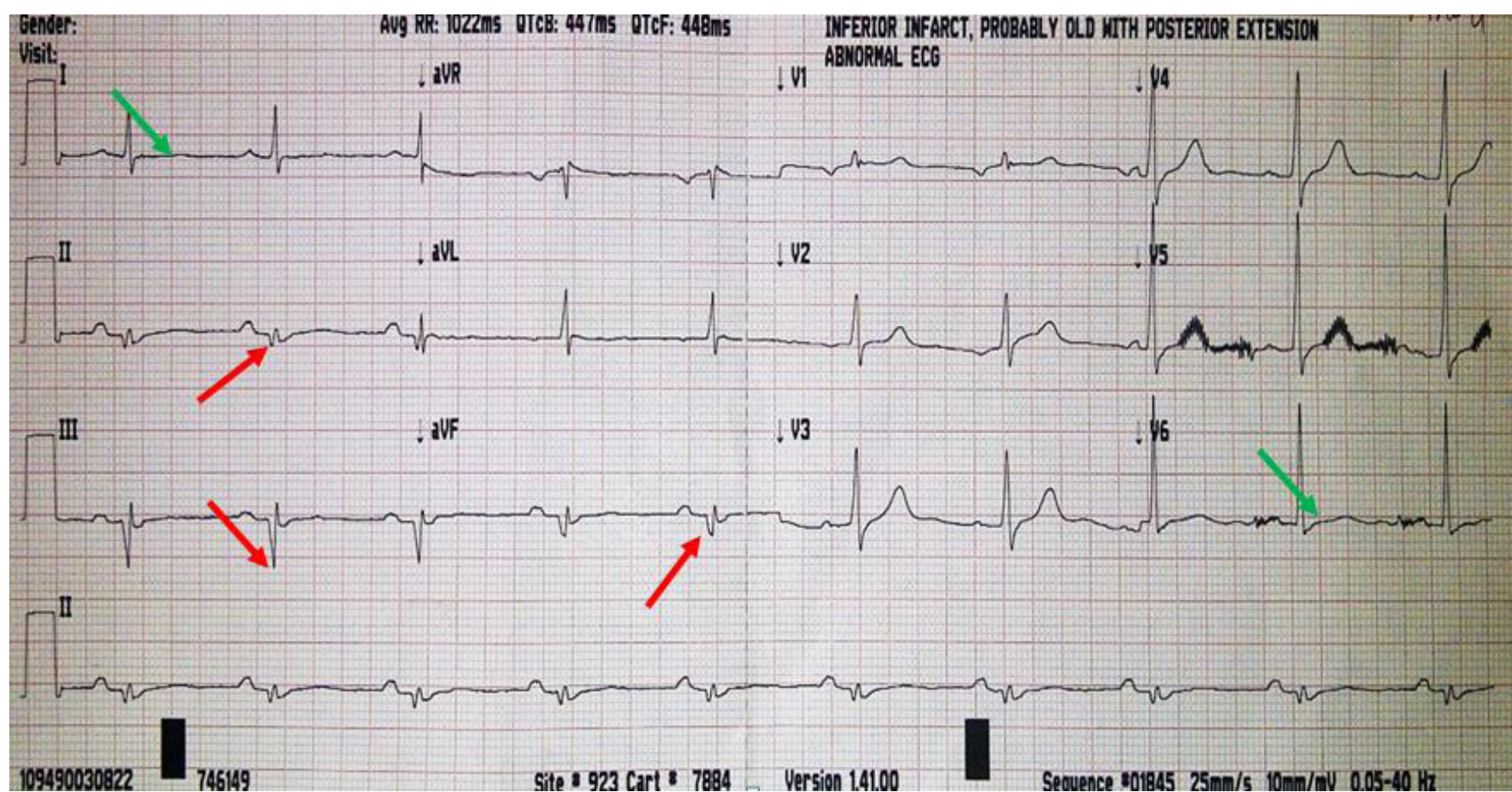

Fig. 1. ECG at admission showing pathological $Q$ waves in the inferior leads, flat T waves in DI, DII, DIII and V6

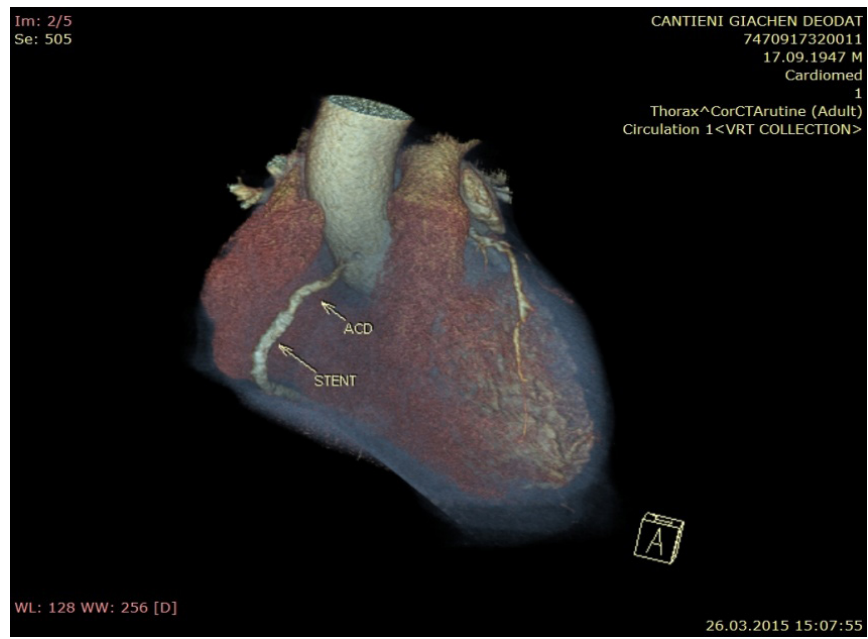

Fig. 2. Angio CT Multislice showing patent right coronary artery

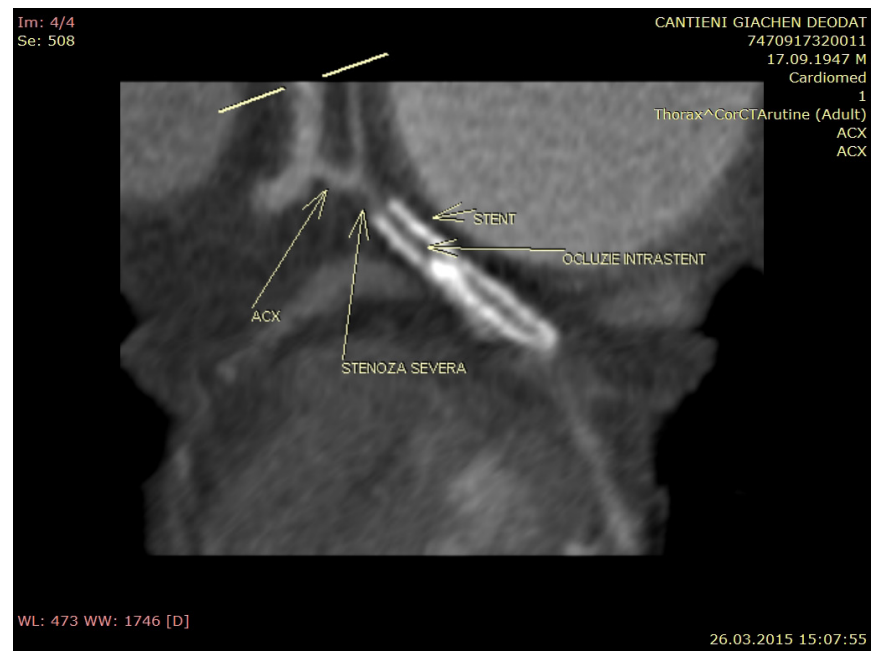

Fig. 4. Angio CT revealing occlusion of the coronary stent

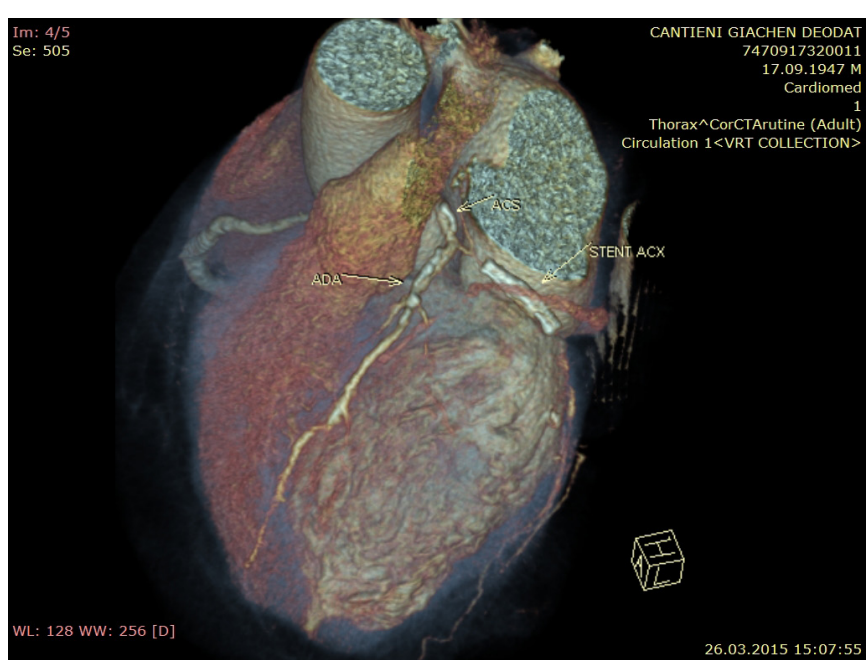

Fig. 3. Angio CT Multislice showing occlusion of the circumflex artery

rapidly retrograde to the left main (fig. 5), causing hemodynamic instability, with a significant drop in the blood pressure and signs of cardiogenic schock. The patient received urgent inotropic support and the rapid response of the interventional cardiologist was to implant a stent in the left coronary artery trunk (figure 6). Inotropic support (Dopamine $5 \mathrm{ug} / \mathrm{kg} / \mathrm{min}$ ) was maintained for 6 hours postintervention to maintain an optimal blood pressure. Subsequently, the control angiography revealed a stent well apposed to the coronary artery wall, resolving the dissection (Figure 7), and the patient was hemodynamically stable, without any angina.

\section{Discussions}

Ischemic complications have been reported in approximately $4 \%$ of patients undergoing coronary angioplasty 


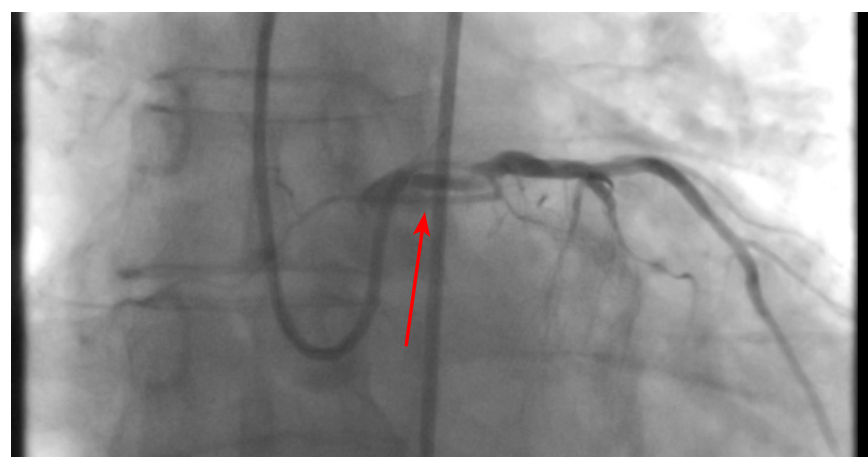

Fig. 5. Coronary angiography indicating a dissection localized proximal to the stent

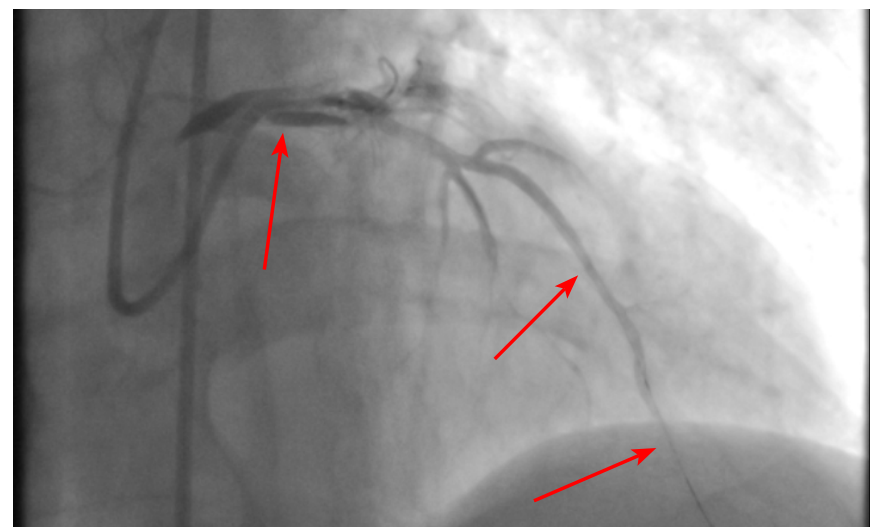

Fig. 6. Attempt to cross the lesion with a guidewire, causing coronary dissection transmitted retrogradely into the left main

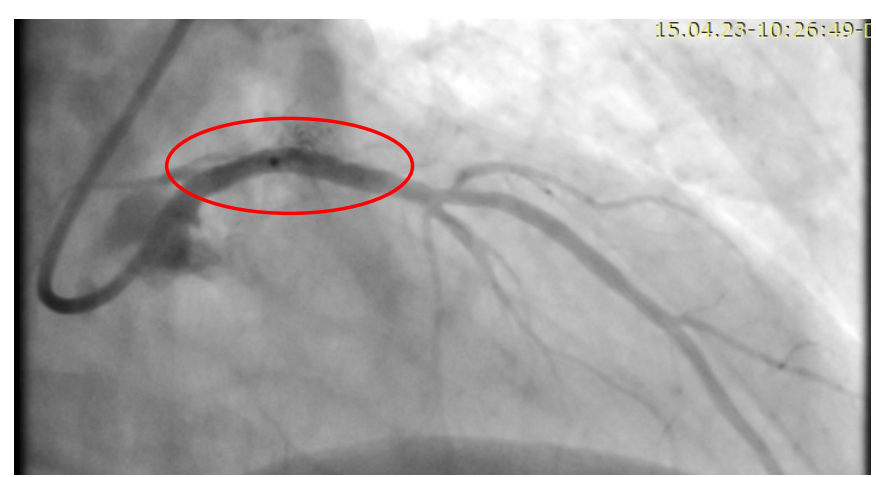

Fig. 7. Optimal post PTCA result after stent implantation

and in about $2 \%$ after leaving the catheterization laboratory [3]. Although spontaneous coronary artery dissection is considered to predominately affect young individuals, most previous studies have shown otherwise, with the higher prevalence in the 5th/6th decade of life [4]. Coronary angiography is the most widely used diagnostic technique for visualisation of the coronary arteries
[5]. Angiography, however, is unable to visualize the vessel wall and has a limited diagnostic accuracy. In this setting, novel tomographic techniques, such multislice computed tomography (MSCT), provide unique diagnostic insights [6] for a better preparation of the procedure. Despite the certain advantages provided by Angio CT examination and despite the proper preparation of the patient, in this casethe patient presented a left coronary dissection resulting from the attempt to cross the circumflex artery occlusion with the BMW guide. As in other studies found in the medical literature $[3,7]$, the operator urgently implanted a coronary stent, with very good angiographic results, which saved patient's life.

\section{Conclusions}

Coronary artery dissection is a very serious complication that can occur during a complex revascularization procedure, requiring immediate intervention in order to save thepatient's life.

\section{Acknowledgement}

This paper is supported by the Sectoral Operational Programme Human Resources Development (SOP HRD), financed from the European Social Fund and by the Romanian Government under the contract number POSDRU/159/1.5/S/133377/

\section{Conflict of interest}

None to declare.

\section{References}

1. Pretty HC. Dissecting aneurysm of coronary artery in a woman aged 42:rupture. BMJ 1931;1:667.

2. Forker A.D., Rosenlof R.C., Weaver W.F., Carveth S.W. Reese H.E. Primary dissecting aneurysm of the right coronary artery with survival. Chest. 1973;64:656-658.

3. Ellis SG, Roubin GS, King SB III, et al. Angiographic and clinical predictors of acute closure after native vessel coronary angioplasty. Circulation 1988;77:372

4. Motreff P., Souteyrand G., Dauphin C., et al. Management of spontaneous coronary artery dissection: review of the literature and discussion based on a series of 12 young women with acute coronary syndrome. Cardiology. 2010;115:10-18.

5. Vrints C.J.; Spontaneous coronary artery dissection. Heart. 2010;96:801-808.

6. Alfonso F., Paulo M., Gonzalo N., Diagnosis of spontaneous coronary artery dissection by optical coherence tomography. J Am CollCardiol. 2012;59:1073-1079.

7. PJ de Feyter, M van den Brand, GJ Laarman, et al. Acute coronary artery occlusion during and after percutaneous transluminal coronary angioplasty: frequency, prediction, clinical course, management, and follow-up Circulation. 1991;83,pp.927-936. 